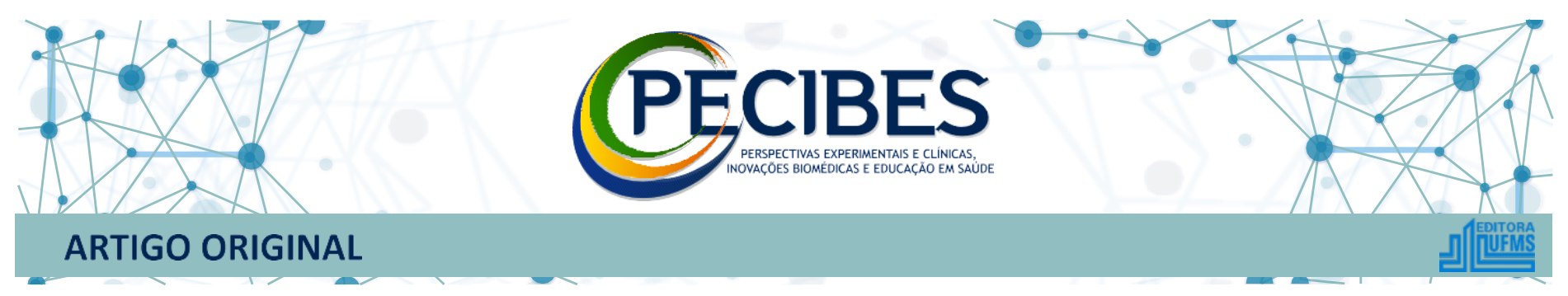

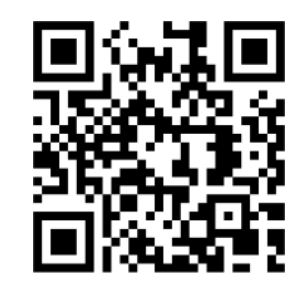

http://www.seer.ufms.br/index.php/p ecibes/index

*Autor correspondente: Isabela Alves de Souza, Hospital Regional de Mato Grosso do Sul - HRMS.

E-mail do autor: isa_julie@ hotmail.com

Palavras-chave: Sepse. Adesão plaquetária. Ativação plaquetária. Agregação plaquetária. COVID-19.

Key-words: Sepsis. Platelet adhesion. Platelet activation. Platelet aggregation. COVID-19.

\section{Avaliação da função plaquetária em pacientes sépticos internados em unidades de terapia intensiva durante a pandemia de COVID-19.}

Evaluation of platelet function in septic patients hospitalized to intensive care units during the COVID-19 pandemic.

Souza, Isabela A. ${ }^{1}$, Cruz, Letícia A. ${ }^{1}$, Correia, Rodolfo P. ${ }^{2}$, Almeida, Eliane B. ${ }^{1}$.

${ }^{1}$ Laboratório de Análises Clínicas (LAC), Hospital Regional de Mato Grosso do Sul (HRMS). ${ }^{2}$ Célula Diagnósticos Citológicos, Campo Grande, MS.

Resumo

Introdução: A linhagem das plaquetas tem sido amplamente investigada na sepse, uma vez que anormalidades no sistema hemostático são eventos comuns nesses pacientes. O objetivo deste estudo foi avaliar a adesão, ativação e agregação plaquetária em pacientes sépticos internados em Unidades de Terapia Intensiva durante a pandemia de COVID-19 por meio da expressão de marcadores de membrana plaquetária. Método: A função plaquetária foi investigada em 36 pacientes sépticos de início recente e 8 controles de Junho a Setembro de 2020 em um grande hospital público. Foi obtido plasma rico em plaquetas, marcado com os anticorpos monoclonais: CD42b (adesão), CD36 (ativação) e CD41 e CD61 (agregação), e análise em citometria de fluxo para obtenção da expressão dos marcadores em intensidade mediana de fluorescência (IMF). O software GraphPad InStat@ foi utilizado para análise estatística e o valor de $\mathrm{p}<0,05$ foi considerado estatisticamente significativo. Principais resultados: Para os quatro marcadores analisados, a IMF foi menor nos pacientes sépticos do que nos controles, mas houve diferença estatisticamente significativa apenas na IMF de CD41 e CD36 ( $\mathrm{p}<0,05)$. Trinta e um (31) pacientes sépticos $(86 \%)$ foram positivos para COVID-19, mas não houve diferença estatística $(\mathrm{p}>0,05)$ entre as IMFs de positivos e negativos. Conclusão: A sepse diminui a função hemostática das plaquetas circulantes, principalmente na ativação e agregação. Esse fenômeno pode ser decorrente da resposta inflamatória, onde antes do consumo e exaustão das plaquetas, ocorre uma reorganização da função plaquetária para conter o dano hemostático da formação generalizada de coágulos, comum na sepse, e característica marcante da COVID-19.

\section{Abstract}

Background: The platelet lineage has been widely investigated in sepsis, since abnormalities in the haemostatic system are common events in these patients. The aim of this study was to evaluate adherence, activation and platelet aggregation in septic patients admitted to Intensive Care Units during the COVID-19 pandemic through the expression of platelet membrane markers. Method: Platelet function was investigated in 36 septic patients with recent onset and 8 controls from June to September 2020 in a large public hospital. Platelet-rich plasma was obtained, labeled with monoclonal antibodies: CD42b (adhesion), CD36 (activation) and CD41 and CD61 (aggregation), and analysis in flow cytometry to obtain the expression of the markers at median fluorescence intensity (MFI). The GraphPad InStat $\subset$ software was used for statistical analysis and the value of $p<0,05$ was considered statistically significant. Main results: For the four markers analyzed, the MFI was lower in septic patients than in controls, but there was only a statistically significant difference in the MFI of CD41 and CD36 ( $<<0,05)$. Thirty-one (31) septic patients (86\%) were positive for COVID-19, but there was no statistically difference $(\mathrm{p}>0,05)$ between positive and negative MFIs. Conclusion: Sepsis decreases the haemostatic function of circulating platelets, mainly in activation and aggregation. This phenomenon may be the result of the inflammatory response, where before the consumption and exhaustion of platelets, there is a reorganization of platelet function to contain the haemostatic damage of the generalized formation of clots, common in sepsis, and a striking characteristic of COVID-19. 


\section{Introdução}

A sepse é um problema de saúde pública, responsável por altos índices de internações e mortalidade em unidades de terapia intensiva (Zonta et al., 2018). De acordo com o estudo SPREAD (Sepsis Prevalence Assessment Database) em 227 UTIs brasileiras, a prevalência e a mortalidade de pacientes com sepse é de $30,2 \%$ e $55,7 \%$, respectivamente (Machado et al., 2017).

A linhagem das plaquetas tem sido extensivamente pesquisada na sepse, uma vez que anormalidades no sistema hemostático são eventos comuns nesses pacientes, variando de pequenas alterações na contagem de plaquetas e testes de coagulação ao desenvolvimento de coagulação intravascular disseminada e trombose microvascular generalizada, que refletem significativamente na clínica do paciente (Valele-Jiménez et al., 2018).

Com o advento da COVID-19, também foram observados sepse e choque séptico por infecção por SARSCoV-2, que acredita-se ser capaz de atacar diretamente as células endoteliais vasculares por expressarem níveis elevados da enzima de conversão da Angiotensina2 (ACE2), cujo receptor é usado pelo vírus para entrar nas células hospedeiras, o que pode levar a uma coagulação anormal ( $\mathrm{Li}$ et al., 2020).

Diversas patologias associadas à disfunção plaquetária podem ser avaliadas pela expressão de diferentes marcadores imunofenotípicos por citometria de fluxo, que é cada vez mais utilizada para a caracterização específica de alterações fenotípicas em plaquetas relacionadas à adesão, ativação e agregação por meio da identificação de diferentes antígenos plaquetários com o uso de anticorpos monoclonais específicos (Cecconello, 2015).

Dentre as várias glicoproteínas de membrana específicas associadas às plaquetas, destacamos a GPIb (CD42b), pertencente ao complexo responsável pela adesão, GPIIIb (CD36) pela ativação e GPIIb (CD41a) e GPIIIa (CD61) pela agregação plaquetária (Koupenova et al., 2018).

Mesmo com o avanço das terapias antimicrobianas e vasopressoras, o sucesso terapêutico na sepse também está intimamente ligado à hemostasia, exigindo dados e ferramenas que colaborem com o trabalho da equipe no cuidado, incluindo o conhecimento da função plaquetária na sepse, campo ainda pouco explorado na literatura, principalmente na sepse por SARS-CoV-2 (Akinosoglou et al., 2017; Cecconi et al., 2018; Maiese et al., 2020).

Nesse contexto, avaliamos a adesão, ativação e agregação plaquetária em pacientes com sepse por meio da expressão de seus principais marcadores de membrana, buscando compreender melhor a atividade e os mecanismos envolvidos na fisiopatologia da doença.

\section{Material e Métodos}

\subsection{Desenho do estudo}

Este estudo prospectivo observacional analítico foi realizado no período de Junho a Setembro de 2020 no Hospital Regional de Mato Grosso do Sul (HRMS). Este estudo foi aprovado pelo comitê de ética do HRMS conforme número 56/2019 e pelo comitê de ética da Universidade Anhanguera Uniderp (CAAE 28609020.1.0000.5161). Dada a natureza do estudo, os pacientes ou familiares assinaram o termo de consentimento livre e esclarecido.

\subsection{População estudada}

A função plaquetária foi investigada em 36 pacientes sépticos de início recente admitidos em UTI, a maioria deles internados por COVID-19, e em 8 controles sem sepse. Os pacientes sépticos foram definidos e classificados em sepse ou choque séptico pela equipe médica com base nas definições do Terceiro Consenso Internacional para Sepse e Choque Séptico (Sepsis-3) (Singer et al., 2016). Os critérios de exclusão foram: idade inferior a 18 anos; e indivíduos com qualquer doença hematológica, para evitar qualquer potencial interferência nos resultados. Os controles sem sepse não tinham evidência de infecção.

A triagem dos pacientes foi realizada por meio de consulta ao prontuário eletrônico. Foram coletados idade, sexo, contagem global de leucócitos, dosagem de hemoglobina, proteína $\mathrm{C}$ reativa (PCR), dímero $\mathrm{D}$, RDW, contagem de plaquetas, resultado para COVID-19 e hemocultura. A gravidade da doença foi avaliada de acordo com o escore APACHE II e o desfecho foi classificado como alta ou óbito 28 dias após o diagnóstico.

\subsection{Obtenção de plasma rico em plaquetas e estruturas de marcação}

Após o diagnóstico de sepse pela equipe médica, as amostras de sangue total em tubo de EDTA da rotina diária das Unidades de Terapia Intensiva (UTI) foram centrifugadas a 800 rotações por minuto (rpm) por 10 minutos para separação do plasma rico em plaquetas (PRP) dos outros componentes celulares. O PRP foi transferido para outro tubo usando uma pipeta Pasteur. Em seguida, $60 \mu 1$ de PRP foram incubados com $1200 \mu \mathrm{l}$ de paraformaldeído a $1 \%$ por 10 minutos em temperatura ambiente em um tubo Falcon. Posteriormente, $2,5 \mathrm{ml}$ de solução salina de tampão fosfato (PBS) foram adicionados e as amostras foram centrifugadas durante 15 minutos a $1800 \mathrm{rpm}$. O sobrenadante foi então descartado e a amostra ressuspensa em $180 \mu \mathrm{l}$ de PBS. Foram distribuídos $45 \mu \mathrm{l}$ desta suspensão em 4 tubos acrílicos e adicionado os anticorpos monoclonais de acordo com o mapa de trabalho: CD41a - FITC (Glicoproteína IIb), CD42b - PE (Glicoproteína Ib), CD36 - FITC (Glicoproteína IIIb) e CD61 - FITC (Glicoproteína IIIa), todos da Becton Dickinson (BDC) (Nova Jersey, Estados Unidos); e incubou-se por 30 minutos à temperatura ambiente, protegidos da luz. Posteriormente, acrescentou-se $2 \mathrm{ml}$ de PBS e centrifugou-se por 10 minutos a $1800 \mathrm{rpm}$. O sobrenadante foi então desprezado e a amostra ressuspensa em $500 \mu \mathrm{l}$ de PBS para análise no citômetro de fluxo em até 72 horas (Adaptado de Ferreira, 2009). 


\subsection{Análise por citometria de fluxo}

Foram adquiridos 50.000 eventos no citômetro de fluxo (CytoFLEX Flow Cytometer@, Beckman Coulter), selecionando a população de plaquetas no plot FSCXSSC (TamanhoxGranularidade) em escala logarítmica. Os dados foram analisados no software Kaluza $\odot$ (Beckman Coulter)para obtenção da intensidade mediana de fluorescência dos marcadores plaquetários. A intensidade de fluorescência foi analisada no filtro de 488 $\mathrm{nm}$.

\subsection{Análise estatística}

Todos os valores são expressos como mediana e intervalo de confiança. Na comparação entre variáveis paramétricas foi utilizado o teste t de Student não pareado e para variáveis não paramétricas o teste de Mann-Whitney. Para análise das correlações entre as variáveis foi utilizado $o$ teste de Spearman. Valores de $\mathrm{p}<0,05$ foram considerados significativos. Todas as análises foram realizadas no software GraphPad InStat@.

\section{Resultados}

Participaram deste estudo 36 pacientes, 4 (11\%) apresentaram sepse e $32 \%$ (89\%) desenvolveram choque séptico, com média de idade de $66 \pm 12$ anos, a idade mínima encontrada foi de 38 anos e a máxima de 87 anos. O grupo controle inclui 5 mulheres e 3 homens com idade média de $39 \pm 17$ anos e contagem de plaquetas dentro do valor de referência $\left(150.000-400.00 \mathrm{~mm}^{3}\right)$. As características dos pacientes são apresentadas nas Tabelas 1,2 e 3 e a comparação das intensidade de fluorescência entre pacientes sépticos e controles na Tabela 4.

A contagem de leucócitos global dos pacientes sépticos variou de $4.530 \mathrm{~mm}^{3}$ a $37.7000 \mathrm{~mm}^{3} \mathrm{com}$ mediana de $14.180 \mathrm{~mm}^{3} ; 28(78 \%)$ apresentaram leucocitose (acima de $\left.10.000 \mathrm{~mm}^{3}\right), 6(16 \%)$ obtiveram contagem de leucócitos dentro do valor de referência (5.000-10.000 $\left.\mathrm{mm}^{3}\right)$ e $2(6 \%)$ apresentaram leucopenia (abaixo de 5.000 $\left.\mathrm{mm}^{3}\right)$.

Na série vermelha, as dosagens de hemoglobina variaram de $6,3 \mathrm{~g} / \mathrm{dL}$ a $15,8 \mathrm{~g} / \mathrm{dL}$ com mediana de 9,6 g/dL; $26(72 \%)$ apresentaram algum grau de anemia (Hemoglobina inferior a $12 \mathrm{~g} / \mathrm{dL}$ ) e anisocitose (RDW superior a $14 \%)$ e $10(28 \%)$ tiveram dosagens dentro do valor de referência (acima de $12 \mathrm{~g} / \mathrm{dL}$ ) sem anisocitose.

A contagem de plaquetas variou de $105.000 \mathrm{~mm}^{3}$ a $603.000 \mathrm{~mm}^{3}$ com mediana de $236.000 \mathrm{~mm}^{3}$. Trinta (30) pacientes sépticos (83\%) obtiveram contagem de plaquetas dentro do valor de referência, $4(11 \%)$ apresentaram trombocitopenia (abaixo de $150.000 \mathrm{~mm}^{3}$ ) e $2(6 \%)$ apresentaram trombocitose (acima de 400.000 $\left.\mathrm{mm}^{3}\right)$.
Tabela 1 - Características dos pacientes sépticos de acordo com o sexo (n e \%; média e SD; mediana e intervalo de confiança).

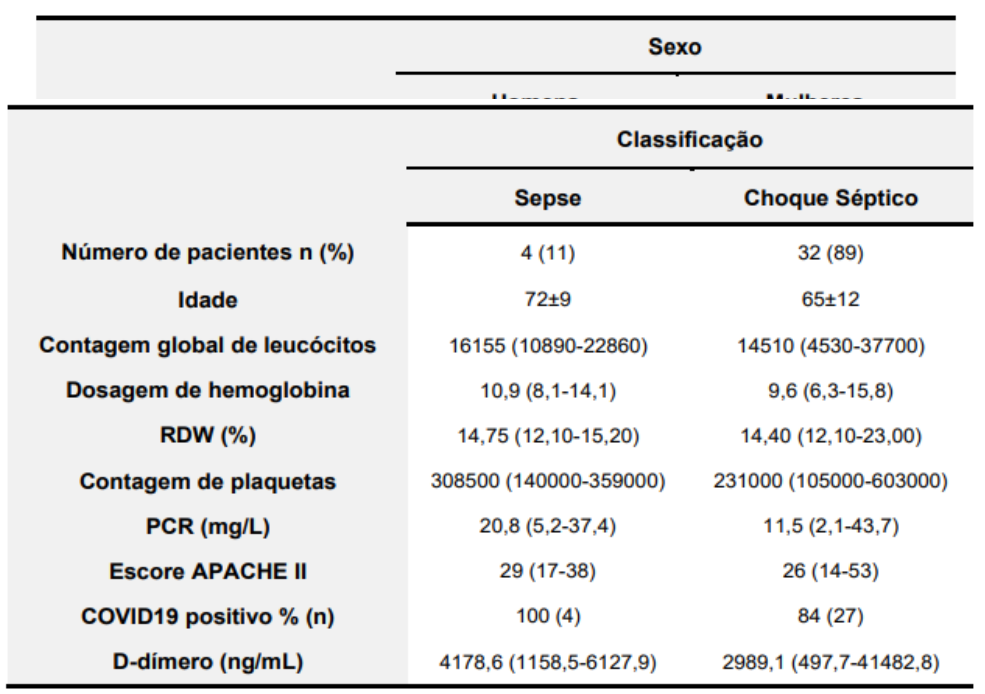

Tabela 2 - Características dos pacientes sépticos de acordo com a classificação (n e \%; média e SD; mediana e intervalo de confiança).

Tabela 3 - Características dos pacientes sépticos de acordo com o desfecho (n e \%; média e SD; mediana e intervalo de confiança).

\begin{tabular}{ccc}
\hline & \multicolumn{2}{c}{ Desfecho } \\
\cline { 2 - 3 } & Alta & Óbito \\
\cline { 2 - 3 } Idade & $14(39)$ & $22(61)$ \\
Número de pacientes n (\%) & $66 \pm 14$ & $67 \pm 11$ \\
Contagem global de leucócitos & $12585(4600-19770)$ & $15315(4530-37700)$ \\
\hline RDW (\%) & & \\
Dosagem de hemoglobina & $10,9(6,3-15,8)$ & $9,6(6,8-14,0)$ \\
Contagem de plaquetas & $14,25(12,10-21,70)$ & $14,60(12,10-23,00)$ \\
PCR (mg/L) & $204500(105000-603000)$ & $243500(147000-407000)$ \\
Escore APACHE II & $8,8(2,1-41,7)$ & $12,3(3,5-43,7)$ \\
CoVID19 positivo $\mathbf{n}(\%)$ & $26(17-53)$ & $28(14-40)$ \\
D-dimero (ng/mL) & $10(71)$ & $21(95)$ \\
\hline
\end{tabular}

Tabela 4 - Comparação das intensidades medianas de fluorescência dos pacientes sépticos e controles para os marcadores estudados em citometria de fluxo (mediana e intervalo de confiança).

\begin{tabular}{lccc}
\hline & Controles $(\mathrm{n}=8)$ & Pacientes sépticos $(\mathrm{n}=36)$ & Valor de $\mathbf{p}$ \\
\hline CD41 $^{*}$ & $3.754(2.684-8.133)$ & $2.376(0.847-8.604)$ & 0,012 \\
CD42 & $16.529(10.441-35.478)$ & $11.884(0.920-30.189)$ & 0,211 \\
CD36 $^{*}$ & $19.326(9.254-24.928)$ & $10.484(1.610-22.300)$ & 0,011 \\
CD61 & $33.068(8.367-48.592)$ & $29.510(6.796-45.974)$ & 0,558 \\
\hline${ }^{*} \mathrm{p}<0,05$ pacientes sépticos vs. controles; & &
\end{tabular}

Quanto a PCR, as dosagens variaram de $2,1 \mathrm{mg} / \mathrm{L}$ a $43,7 \mathrm{mg} / \mathrm{L}$ com mediana de $11,5 \mathrm{mg} / \mathrm{dL} ; 27$ pacientes sépticos $(75 \%)$ apresentaram dosagens elevadas (acima de $6 \mathrm{mg} / \mathrm{L}$ ) e 9 $(25 \%)$ tiveram dosagens dentro do valor de referência (inferior 
a $6 \mathrm{mg} / \mathrm{dL})$.

Já as dosagens de D-dímero variaram de 497,7 $\mathrm{ng} / \mathrm{mL}$ a $159.655,6 \mathrm{ng} / \mathrm{mL}$ com mediana de $3.319,8 \mathrm{ng} / \mathrm{mL}$. Trinta e cinco (35) pacientes sépticos (97\%) tiveram dosagens altas (acima de $500 \mathrm{ng} / \mathrm{mL}$ ) e apenas 1 (3\%) dentro do valor de referência (abaixo de $500 \mathrm{ng} / \mathrm{mL}$ ).

Quanto ao isolamento do microrganismo causador da sepse, em nosso estudo, dos 36 pacientes, 24 (67\%) tiverhemoculturas negativas, 9 (25\%) tiveram hemoculturas positivas e $3(8 \%)$ não tiveram pedidos de hemocultura solicitados. Os microrganismos isolados foram Staphylococcus sp. $(\mathrm{n}=7)$, Corynebacterium $s p .(\mathrm{n}=1)$ e Enterococcus $s p .(\mathrm{n}=1)$.

Quanto ao diagnóstico de COVID-19, foram considerados positivos os pacientes que apresentaram resultado "Detectável" pela metodologia Reação da Cadeia da Polimerase em Tempo Real (RT-PCR) e/ou resultado "Reagente" no teste imunocromatográfico, conhecido como teste rápido. Em nosso estudo, 31 pacientes sépticos $(86 \%)$ eram positivos para COVID-19 e 5 (14\%) eram negativos. Quando comparadas as intensidades medianas de fluorescência e a contagem de plaquetas entre os COVID-19 positivos e negativos, não houve diferença estatística significativa.

No escore APACHE II, que estima o risco de mortalidade, 9 pacientes sépticos (25\%) fizeram de 25-29 pontos, o que corresponde a 55\% de chance de morte, $7(19 \%)$ marcaram 35-100 pontos (85\% de chance de morte), 5 (14\%) pontuaram 30-34 pontos ( $73 \%$ de chance de morte), 9 (25\%) fizeram 20-24 pontos (40\% de chance de morte), 4 (11\%) marcaram $15-19$ pontos ( $24 \%$ de chance de morte) e $1(3 \%)$ fez 10-14 pontos (15\% de chance de morte). O escore não foi aplicado em 1 paciente (3\%) do estudo. Na comparação entre a população no escore APACHE e o desfecho dos pacientes não houve diferença significativa.

As correlações significativas das variáveis dos pacientes sépticos são apresentadas na Tabela 5. Houve correlações negativas ente PCR e CD41, PCR e CD61, APACHE e contagem de plaquetas e dosagem de Dímero-D e CD41; e correlação positiva entre APACHE e CD42. Não houve correlação significativa entre as intensidades medianas de fluorescência e a contagem de plaquetas, e Dímero-D e contagem de plaquetas.

Tabela 5 - Grau de correlação das variáveis dos pacientes sépticos.

\begin{tabular}{cccc}
\hline Variáveis & Significância $(\mathbf{p})$ & Coeficiente $(\mathbf{r})$ & Correlação* $^{\star}$ \\
\hline PCR e CD41 & 0,0218 & $-0,3812$ & Fraca \\
PCR e CD61 & 0,0193 & $-0,3883$ & Fraca \\
APACHE e CD42 & 0,0496 & $+0,3344$ & Fraca \\
APACHE e Plaquetas & 0,0069 & $-0,4481$ & Moderada \\
Dímero D e CD41 & 0,0009 & $-0,5515$ & Moderada \\
\hline
\end{tabular}

${ }^{*} \mathrm{r}$ (+ ou -) 0,00 a 0,19 (correlação bem fraca), 0,20 a 0,39 (correlação fraca), 0,40 a 0,69 (correlação moderada), 0,70 a 0,89 (correlação forte), 0,90 a 1,00 (correlação muito forte).

\section{Discussão}

Em tempos de pandemia de COVID-19, verificamos que o perfil de pacientes que apresentaram sepse de início recente e estavam internados em unidades de terapia intensiva em nosso estudo eram homens (72\%), acima de 65 anos, em choque séptico (89\%). Ao diagnóstico, apresentavam leucocitose (78\%), por volta de 14.000 leucócitos $/ \mathrm{mm}^{3}$, anemia entre 9 e $11 \mathrm{~g} / \mathrm{L}$ com anisocitose e plaquetas dentro da normalidade (83\%). Os níveis de PCR e D-dímero estavam elevados (75\% e 97\%, respectivamente), com hemoculturas negativas (67\%) e eram positivos para COVID-19 (86\%). Na entrada da unidade, pontuaram APACHE II com 40 a $55 \%$ de chance de morte e $61 \%$ vieram a óbito.

O desfecho desfavorável encontrado em nosso estudo pode ser correlacionado com vários fatores potenciais de mau prognóstico no estágio inicial da sepse, como a idade avançada, leucocitose, alteração nos parâmetros de coagulação sanguínea com o exemplo do D-dímero, maior que $1.000 \mathrm{ng} / \mathrm{mL}$, e os marcadores inflamatórios elevados (PCR) (Maiese et al., 2020).

Consta na literatura que adultos com idade avançada (maiores de 60 anos) constituem uma população com risco significativamente maior em ter sepse pela maior incidência de comorbidades, tempo de hospitalização prolongados e imunossenescência, fatores que também aumentam a suscetibilidade à infecção viral e, portanto, á sepse viral (Lin et al., 2018). Esta predisposição foi reafirmada em nosso estudo, onde a média de idade dos pacientes sépticos foi maior que 65 anos.

A função plaquetária envolve os processos de adesão, ativação e agregação. No presente estudo, avaliamos cada etapa por meio da expressão dos marcadores de membrana correspondnetes e conforme Tabela 4, não houve diferença estatística significativa na intensidade mediana de fluorescência (IMF) do CD42, pertencente ao complexo responsável pela adesão, entre os pacientes sépticos e controles $(\mathrm{p}=0,21)$, apesar de a mediana da IMF dos pacientes sépticos ter sido menor que dos controles. Yaguchi et al. (2004) não observaram diferença estatística entre os dois grupos.

Verificamos uma correlação positiva ( $\mathrm{p}=0,0496$; $\mathrm{r}=0,3344$ ) entre a pontuação do escore APACHE e a expressão de $\mathrm{CD} 42$, onde quanto maior o risco de mortalidade, maior a IMF de CD42 do paciente. No estudo de Yaguchi et al. (2004), as plaquetas preservaram a expressão do complexo de adesão independentemente da gravidade da sepse. Desta forma, verificamos que a capacidade de adesão das plaquetas é preservada em pacientes sépticos, mesmo que a expressão seja diminuída, e está relacionada à gravidade.

Já a IMF de CD36, correspondente a ativação plaquetária, foi significativamente menor em pacientes sépticos do que nos controles $(\mathrm{p}=0,011)$ em nosso estudo. No estudo de Laursen et al. (2020), a ativação das plaquetas foi significativamente maior em pacientes com choque séptico do que em controles saudáveis. Entretanto, no estudo de Laursen foi utilizado como marcador de ativação plaquetária o CD63 (relacionado ao fibrinogênio ligado), já neste estudo, utilizamos o CD36 (relacionado ao fibrinogênio solúvel). De acordo com a literatura, uma explicação para a discrepância entre nossos dados pode estar relacionada a uma pré-ativação das plaquetas quando submetidas aos procedimentos que preparam a amostra para a análise e a própria demora no processamento no citômetro de fluxo, o que pode potencialmente influenciar os resultados quanto ao aumento 
da ativação plaquetária (Cecconello, 2015; Muronoi et al., 2016).

Quanto à agregação, a IMF de CD41 dos pacientes sépticos foi menor que nos controles, sendo esta diferença estatisticamente significativa $(\mathrm{p}=0,0122)$, porém não se reproduziu em CD61 ( $\mathrm{p}=0,558)$. Yaguchi et al. (2004) observaram diminuição da agregação plaquetária, porém com alteração dos dois marcadores relacionados mesmo em pacientes sépticos com contagem de plaquetas dentro da normalidade e independente dos níveis de D-dímero, mas sim relacionada à gravidade da sepse, sugestivo de alteração no nível da via da transdução intracelular. Laursen et al. (2020) também observaram agregaçã plaquetária significativamente menor em pacientes com choque séptico do que em controles saudáveis ( $\mathrm{p}<0,0001)$, assim como Brenner et al., 2012. Em nosso estudo, houve correlação negativa entre as dosagens de D-dímero e a expressão de CD41, onde quanto maiores os níveis de D-dímero, menor a expressão de CD41, sugerindo uma regulação negativa dos receptores de superfície para reduzir a produção de coágulos, comuns tanto na sepse, como na COVID-19.

Excluímos pacientes com doenças hematológicas, que normalmente apresentam disfunções plaquetárias, para evitar qualquer potencial interferência nos resultados, porém não podemos evitar o tratamento com antibióticos, corticoides e anticoagulantes, conhecidos por influenciarem na agregação plaquetária, já que a maior parte de nossos pacientes (86\%) eram positivos para COVID-19. Dos 36 pacientes sépticos, $34(94 \%)$ possuíam anticoagulante prescrito, e dois $(6 \%)$ possuíam relato de agregado plaquetário no hemograma. Apenas dois tipos de anticoagulantes foram utilizados pelos pacientes, Enoxaparina Sódica (79\%) e Heparina Sódica (21\%). Segundo a bula da Enoxaparina Sódica, nas doses utilizadas na prevenção de trombose venosa, o fármaco não influencia significativamente na agregação plaquetária.

Sabemos que a sepse pode ser causada por uma ampla gama de patógenos, porém as infecções bacterianas representam a maior parte dos casos e o diagnóstico de sepse viral permanece raro, ficando em evidência apenas agora com a pandemia de COVID-19, apesar de estar associada a maiores influências de disfunção orgânica aguda e mortalidade (Assinger et al., 2019).

Segundo Lin e colaboradores (2018), até $42 \%$ das apresentações de sepse têm hemoculturas negativas, sugerindo uma causa não bacteriana. Em nosso estudo, $67 \%$ das hemoculturas foram negativas. Estes dados reforçam a hipótese da ocorrência da doença pela ação do vírus SARSCoV-2, já que a maioria dos pacientes sépticos estudados que tiveram hemoculturas negativas $(87,5 \%)$ foram diagnosticados com COVID-19, e as garrafas de hemocultura utilizadas para análise possuíam inibidor de antimicrobianos, fato que não impediria o crescimento de um possível patógeno bacteriano. Porém, esta associação pode ser ainda maior, pois entre as hemoculturas positivas, apenas uma era indicativa de real infecção, já que os outros microrganisos isolados são sugestivos de contaminação na coleta.

Concluímos que a sepse diminuiu a função hemostática das plaquetas circulantes, principalmente na ativação e agregação plaquetária. Este fenômeno pode ser resultado da resposta inflamatória, onde antes do consumo e exaustão das plaquetas, há uma reorganização da função plaquetária para conter o prejuízo hemostático da formação generalizada de coágulos, comuns na sepse, e característica marcante da COVID-19. Em algumas etapas, como na adesão, observamos que pacientes mais graves ainda apresentavam maior atuação plaquetária. Porém a função plaquetária foi avaliada apenas ao diagnóstico de sepse, os presentes resultados não podem descrever o desenvolvimento posterior de adesão, ativação e agregação no curso da patologia.

Concluímos também que a sepse, nos pacientes de nosso estudo, foi desencadeada pelo vírus SARS-CoV-2, agente etiológico da COVID-19, já que a maior parte das hemoculturas eram negativas para bactérias e fungos $(67 \%) \mathrm{e}$ as garrafas de hemocultura utilizadas possuíam inibidor de antimicrobianos, reforçando a hipótese da ocorrência de sepse viral. Os pacientes mais acometidos eram homens, acima de 65 anos, com altas dosagens de D-dímero e PCR no início da sepse, que evoluíram para choque séptico com desfecho desfavorável em mais de $60 \%$ dos casos.

Este estudo preliminar colabora com a formação de um retrato do perfil de pacientes sépticos em Unidades de Terapia Intensiva durante a pandemia de COVID-19, suas alterações hemostáticas e a interferência do SARS-CoV-2 nesta patologia, porém são necessáros estudos posteriores para melhores conclusões por se tratar de um assunto recente e ainda em discussão no mundo todo.

\section{Agradecimentos}

Agradecemos aos colaboradores do Hospital Regional de Mato Grosso do Sul, especialmente a equipe do Laboratório de Análises Clínicas, pelo apoio para a realização deste trabalho.

\section{Declaração}

Os autores declaram que o estudo atendeu aos padrões éticos da declaração de Helsinki e a ausência de conflito de interesses.

\section{Referências}

Akinosoglou K, Theodoraki S, Xanthopoulou I, Perperis A, Gkavogianni T, Pistiki A et al. Platelet reactivity in sepsis syndrome: results from the PRESS study. Eur J Clin Microbiol Infec Dis, 36(12), 2503-2512, 2017.

Assinger A, Schrottmaier WC, Salzmann M, Rayes J. Platelets in Sepsis: An Update on Experimental Models and Clinical Data. Front Immunol, 10, 1687, 2019.

Brenner T, Schmidt K, Delang M. Viscoelastic and aggregometric point-of-care testing in patients with septic shock - cross-links between inflammation and haemostasis. Acta Anaesthesiol Scand, 56(10), 2012.

Cecconello DK. Determinação do perfil de expressão dos marcadores imunofenotípicos em plaquetas de doadores do banco de sangue do Hospital de 
Clínicas de Porto Alegre. Monografia (Graduação em Farmácia) - Universidade Federal do Rio Grande do Sul, 2015 .

Cecconi M, Evans L, Levy M, Rhodes A. Sepsis and septic shock. Lancet, 392(7), 75-87, 2018.

Eurofarma. Bula do medicamento Versa®. Disponível em: https://cdn.eurofarma.com.br//wpcontent/uploads/2016/09/Bula_Versa_Paciente.pdf. Acesso em: 29.11.2020. Acesso em: 29/11/2021

Ferreira RP. Investigação de adesão plaquetária na anemia falciforme e o papel dos nucleotídeis cíclicos nesta adesão. Dissertação (Mestrado em Clínica Médica) - Universidade Estadual de Campinas, 2009.

Koupenova M, Clancy L Corkrey HA, Freedman JE. Circulating platelets as mediators of immunity, inflammation and thrombosis. Circ Res, 122(2), 337-351, 2018.

Laursen MA, Larsen JB, Larsen KM, Hvas A-M. Platelet function in patients with septic shock. Thrombosis Research, 185, 33-42, 2020.

Li H, Liu L, Zhang D, Xu J, Dai H, Tang N et al. SARSCoV-2 and viral sepsis: observations and hypotheses. Lancet, 395(10235), 1517-1520, 2020.

Lin G-L, McGinley JP, Drysdale SB, Pollard AJ. Epidemiology and immune pathogenesis of viral sepsis. Front Immunol, 9(2147), 1-21, 2018.

Machado FR, Cavalcanti AB, Bozza FA, Ferreira EM, Carrara FSA, Sousa Jl et al. The epidemiology of sepsis in Brazilian intensive care units (Sepsis Prevalence Assessment Database, SPREAD): an observational study. The Lancet Infectious Diseases, 17(11), 1180-1189, 2017.

Maiese A, Passaro G, Matteis A, Fazio V, Raffaele LR, Paolo M. Thromboinflammatory response in SARS-CoV-2 sepsis. Med Leg J, 88(2), 78-80, 2020.

Muronoi T, Koyama K, Nunomiya S, Lefor AK, Wada $\mathrm{M}$, Koinuma $\mathrm{T}$ et al. Immature platelet function predicts coagulopathy-related platelet consumption and mortality in patients with sepsis. Thromb Res, 144, 169-175, 2016.

Singer M, Deutschman CS, Seymour CW, Shankar-Hari $\mathrm{M}$, Annane $\mathrm{D}$, Bauer $\mathrm{M}$ et al. The Third International Consensus Definitions for Sepsis and Septic Shock (Sepsis-3). JAMA, 315(8), 801810, 2016.

Valle-Jiménez XR, Sánchez-García JC, RevillaRodríguez E, Baltierrez-Hoyos R, Romero- 